\title{
INCORPORATION OF ERROR FUNCTION ABSORPTION COEFFICIENT IN TRANSPORT EQUATION*
}

BY JIN H. CHIN** AND STUART W. CHURCHILL (The University of Michigan)

1. Introduction. The absorption of monochromatic radiation can be represented by the Bouguer-Beer law

$$
T=e^{-\sigma x},
$$

where $T$ is the transmittance, $x$ the path length and $\sigma$ the spectral absorption coefficient. However, absorption varies very rapidly in the region of a band and the average transmittance over a finite interval of wave length in such a region does not follow Eq. (1). The coefficient $\sigma$ cannot then be determined with an instrument of finite resolution. Various models have been proposed for an absorption band in order to derive expressions for the average transmittance. The Elsasser model [1] idealizes an absorption band as an infinite array of equally intense, equidistant lines, each of the Lorentz shape, and each with the same half width. For lines far apart relative to their half-width, this model yields an expression for the "average" transmittance at wave length $\lambda$ which can be condensed to the following form

$$
T_{a}=\operatorname{erfc}(\gamma x)^{1 / 2} .
$$

The error function absorption coefficient $\gamma$ is related to the true spectral coefficient $\sigma$ as follows:

$$
\operatorname{erfc}(\gamma x)^{1 / 2}=\frac{1}{\Delta \lambda} \int_{\lambda-\Delta \lambda / 2}^{\lambda+\Delta \lambda / 2} e^{-\sigma x} d \lambda .
$$

The magnitude of $\Delta \lambda$ must be such that there is no contribution to the transmittance at the center of the interval from lines outside the interval.

Values of $\gamma$ or the equivalent have been determined experimentally for water vapor, carbon dioxide and other gases. In contrast to $\sigma, \gamma$ is a well-behaved function of wave length. For mixtures of two or more independently absorbing gases

$$
\operatorname{erfc}(\gamma x)^{1 / 2}=\operatorname{erfc}\left(\gamma_{1} x\right)^{1 / 2} \cdot \operatorname{erfc}\left(\gamma_{2} x\right)^{1 / 2} \cdot \operatorname{erfc}\left(\gamma_{3} x\right)^{1 / 2} \cdots .
$$

2. Utilization of error function coefficient. The objective of this paper is to demonstrate how the error function absorption coefficient can be incorporated in a solution of the transport equation which describes radiative transfer. Solutions of the transport equation or its equivalent can be expressed in the following general form:

$$
i(\mathbf{R}, \mathbf{\Omega})=F\left(\sigma x, \sigma_{s} x \cdots\right),
$$

where $i$ is the radiant intensity at any position denoted by the vector $\mathrm{R}$ in any direction denoted by the vector $\Omega$. The solution is assumed to be a function of $\sigma x, \sigma_{s} x$ and other parameters, where $x$ is some distance characterizing the position $\mathrm{R}$ and $\sigma$, is the attenuation coefficient for single scattering.

${ }^{*}$ Received May 13, 1959.

**Now with the General Electric Co., Cincinnati, Ohio. 
The parameters and variables other than $\sigma$ are assumed to be constant over a small interval $\Delta \lambda$. The "average" intensity over the interval $\Delta \lambda$ is then

$$
i_{a}=\frac{1}{\Delta \lambda} \int_{\lambda-\Delta \lambda / 2}^{\lambda+\Delta \lambda / 2} i(\mathbf{R}, \boldsymbol{\Omega}) d \lambda=\frac{1}{\Delta \lambda} \int_{\lambda-\Delta \lambda / 2}^{\lambda+\Delta \lambda / 2} F(\sigma x) d \lambda .
$$

This $\lambda$ - integration involving $\sigma x$ can be replaced by the following integral involving $\gamma x$.

$$
\frac{1}{\Delta \lambda} \int_{\lambda-\Delta \lambda / 2}^{\lambda+\Delta \lambda / 2} F(\sigma x) d \lambda=\frac{1}{\pi} \int_{1}^{\infty} \frac{F(\gamma x \eta) d \eta}{\eta\left(\eta-1^{1 / 2}\right)} .
$$

This substitution converts any solution of the transport equation or its equivalent for the monochromatic intensity in terms of $\sigma x$ and other variables and parameters into a new expression for the "average" monochromatic intensity in terms of $\gamma x$ and the other variables and parameters, i.e., Eq. (5) is replaced by

$$
i_{a}(\mathbf{R}, \mathbf{\Omega})=H\left(\gamma x, \sigma_{s} x \cdots\right),
$$

where $H\left(\gamma x, \sigma_{s} x \cdots\right)$ is the function obtained from the integration on the right side of Eq. (7). This integration can be carried out analytically or graphically for a series of values of $\lambda$ depending on the complexity of $F(\sigma x, \cdots)$.

Alternative forms for the integration in terms of the parameter $\gamma$ are

$$
\frac{1}{\Delta \lambda} \int_{\lambda-\Delta \lambda / 2}^{\lambda+\Delta \lambda / 2} F(\sigma x) d x=\frac{1}{\pi} \int_{0}^{1} \frac{F(x \gamma / u) d u}{u^{1 / 2}(1-u)^{1 / 2}}=\frac{2}{\pi} \int_{0}^{\infty} \frac{F\left[\gamma x\left(v^{2}+1\right)\right] d v}{v^{2}+1} .
$$

The theorem represented by Eq. (7) and the two corollaries represented by Eq. (9) are established in the following section.

3. Proof of the theorem and corollaries. Any function sectionally continuous in the interval $(0, \infty)$ may be expanded in terms of the normalized Laguerre polynomials [2]

$$
f(\xi)=\sum_{\nu=0}^{\infty} a_{\nu} \frac{e^{-\xi / 2} L_{\nu}(\xi)}{\nu !},
$$

where $L_{v}(\xi)$ is the Laguerre polynomial of order $\nu$ :

$L_{\nu}(\xi)=e^{\xi} \frac{d^{\nu}}{d \xi^{\prime}}\left(\xi^{\nu} e^{-\xi}\right)=(-1)^{\nu}\left[\xi^{\eta}-\frac{\nu^{2}}{1 !^{\nu}} \xi^{\nu-1}+\frac{\nu^{2}(\nu-1)^{2}}{2 !} \xi^{\nu-2}-\cdots+(-1)^{\nu} \nu !\right]$

and

$$
a_{\nu}=\int_{0}^{\infty} \frac{f(\xi) e^{-\xi / 2} L_{\nu}(\xi) d \xi}{\nu !} .
$$

On physical grounds $F(\sigma x)$ is a continuous or sectionally continuous function of $\sigma x$ and, therefore, can be expanded as follows:

$$
F(\sigma x)=F_{1}(2 \sigma x)=\sum_{\nu=0}^{\infty} \frac{a_{\nu} L_{\nu}(2 \sigma x) e^{-\sigma x}}{\nu !},
$$

where $F_{1}(2 \sigma x)$ is a function of $2 \sigma x$ obtained from the original function $F(\sigma x)$.

Therefore

$$
\frac{1}{\Delta \lambda} \int_{\lambda-\Delta \lambda / 2}^{\lambda+\Delta \lambda / 2} F(\sigma x) d \lambda=\sum_{\nu=0}^{\infty} \frac{a_{\nu}}{\nu !}\left\{\frac{1}{\Delta \lambda} \int_{\lambda-\Delta \lambda / 2}^{\lambda+\Delta \lambda / 2} L_{\nu}(2 \sigma x) e^{-\sigma x} d \lambda\right\},
$$


with

$$
a_{\nu}=2 \int_{0}^{\infty} \frac{F(\xi) L_{\nu}(2 \xi) e^{-\xi}}{\nu !} d \xi .
$$

Since $L_{\nu}(2 \sigma x)$ is a polynomial of argument $2 \sigma x$ and degree $\nu, L_{\nu}(2 \sigma x) e^{-\sigma x}$ can be formed by summation of the products of multiples of powers of $x$ with the differentials of $e^{-\sigma x}$ with respect to $x$. For instance,

$$
\begin{aligned}
& L_{0}(2 \sigma x) e^{-\sigma x}=e^{-\sigma x}, \\
L_{1}(2 \sigma x) e^{-\sigma x}= & (-2 \sigma x+1) e^{-\sigma x}, \\
= & e^{-\sigma x}+(2 x) \frac{d}{d x}\left(e^{-\sigma x}\right), \\
L_{2}(2 \sigma x) e^{-\sigma x}= & {\left[(2 \sigma x)^{2}-4(2 \sigma x)+2\right] e^{-\sigma x}, } \\
= & 4 \sigma^{2} x^{2} e^{-\sigma x}-8 \sigma x e^{-\sigma x}+2 e^{-\sigma x}, \\
= & \left(4 x^{2}\right) \frac{d^{2}}{d x^{2}}\left(e^{-\sigma x}\right)+(8 x) \frac{d}{d x}\left(e^{-\sigma x}\right)+2 e^{-\sigma x},
\end{aligned}
$$

and in general,

$$
\begin{aligned}
L_{\nu}(2 \sigma x) e^{-\sigma x}=(2 x)^{\nu} \frac{d^{\nu}}{d x^{\nu}}\left(e^{-\sigma x}\right)+ & \frac{\nu^{2}}{1 !}(2 x)^{\nu-1} \frac{d^{\nu-1}}{d x^{\nu-1}}\left(e^{-\sigma x}\right) \\
& \quad+\frac{\nu^{2}(\nu-1)^{2}}{2 !}(2 x)^{\nu-2} \frac{d^{\nu-2}}{d x^{\nu-2}}\left(e^{-\sigma x}\right)+\cdots+\nu ! e^{-\sigma x}, \\
= & \sum_{n=0}^{\nu} \frac{(\nu !)^{2}}{[(\nu-n) !]^{2} n !}(2 x)^{\nu-n} \frac{d^{\nu-n}}{d x^{\nu-n}}\left(e^{-\sigma x}\right) .
\end{aligned}
$$

Therefore,

$$
\frac{1}{\Delta \lambda} \int_{\lambda-\Delta \lambda / 2}^{\lambda+\Delta \lambda / 2} F(\sigma x) d \lambda=\sum_{\nu=0}^{\infty} \sum_{n=0}^{\infty} \frac{a_{\nu} \nu !}{[(\nu-n) !]^{2} n !}(2 x)^{\nu-n} \frac{d^{\nu-n}}{d x^{\nu-n}} \cdot\left\{\frac{1}{\Delta \lambda} \int_{\lambda-\Delta \lambda / 2}^{\lambda+\Delta \lambda / 2} e^{-\sigma x} d \lambda\right\} .
$$

The interchange of the order of integration and differentiation is allowable since

$$
\frac{1}{\Delta \lambda} \int_{\lambda-\Delta \lambda / 2}^{\lambda+\Delta \lambda / 2} e^{-\sigma x} d \lambda
$$

exists by definition.

Now erfc $(\gamma x)^{1 / 2}$ is the Laplace transform of $f(t)$ [3], where

$$
f(t)=\left\{\begin{array}{cc}
0 & ; \quad t<\gamma \\
\frac{\gamma^{1 / 2}}{\pi t(t-\gamma)^{1 / 2}} ; & t>\gamma
\end{array}\right.
$$

i.e.,

$$
\operatorname{erfc}(\gamma x)^{1 / 2}=\frac{1}{\pi} \int_{\gamma}^{\infty} \frac{\gamma^{1 / 2} e^{-x t}}{t(t-\gamma)^{1 / 2}} d t
$$


Substituting $t=\gamma \eta$ in the integral, yields

$$
\operatorname{erfc}(\gamma x)^{1 / 2}=\frac{1}{\pi} \int_{1}^{\infty} \frac{e^{-\gamma x \eta}}{\eta(\eta-1)^{1 / 2}} \cdot d \eta .
$$

From Eqs. (3) and (23)

$$
\frac{1}{\Delta \lambda} \int_{\lambda-\Delta \lambda / 2}^{\lambda+\Delta \lambda / 2} e^{-\sigma x} d \lambda=\frac{1}{\pi} \int_{1}^{\infty} \frac{e^{-\gamma x \eta}}{\eta(\eta-1)^{1 / 2}} d \eta .
$$

From Eqs. (20) and (24), interchanging order of integration, differentiation, and summation, yields

$$
\begin{aligned}
\frac{1}{\Delta \lambda} \int_{\lambda-\Delta \lambda / 2}^{\lambda+\Delta \lambda / 2} F(\sigma x) d \lambda & =\sum_{\nu=0}^{\infty} \sum_{n=0}^{\nu} \frac{a_{\nu} \nu !}{[(\nu-n) !]^{2} n !}(2 x)^{\nu-n} \frac{d^{\nu-n}}{d x^{\nu-n}}\left\{\int_{1}^{\infty} \frac{e^{-\gamma x \eta}}{\pi \eta(\eta-1)^{1 / 2}} d \eta\right\} \\
= & \int_{1}^{\infty} \frac{1}{\pi \eta(\eta-1)^{1 / 2}} \sum_{\nu=0}^{\infty} \sum_{n=0}^{\nu} \frac{a_{\nu} \nu !}{[(\nu-n) !]^{2} n !}(2 x)^{\nu-n} \frac{d^{\nu-n}}{d x^{\nu-n}}\left(e^{-\gamma x \eta}\right) d \eta .
\end{aligned}
$$

But, it is evident from Eqs. (13) and (19) that

$$
\sum_{\nu=0}^{\infty} \sum_{n=0}^{\nu} \frac{a_{\nu} \nu !}{[(\nu-n) !]^{2} n !}(2 x)^{\nu-n} \frac{d^{\nu-n}}{d x^{\nu-n}}\left(e^{\gamma x \eta}\right)=F(\gamma \eta x) .
$$

Therefore,

$$
\frac{1}{\Delta \lambda} \int_{\lambda-\Delta \lambda / 2}^{\lambda+\Delta \lambda / 2} F(\sigma x) d \lambda=\frac{1}{\pi} \int_{1}^{\infty} \frac{F(\gamma x \eta) d \eta}{\eta(\eta-1)^{1 / 2}},
$$

and the theorem is proved.

The proof of the corollaries is evident by substituting $\eta=1 / u$ and $\eta=v^{2}+1$ in Eq. (27).

4. Discussion. The incorporation of the error function coefficient in the formal solution of the transport equation immediately permits use of this relatively wellbehaved coefficient in all existing solutions for light scattering, radiant transport, etc., subject only to the validity of the Elsasser model. The resulting "average" intensity, $i_{a}$, can readily be integrated with respect to wave length for any given source distribution to find the total radiation over any finite interval of wave length. These procedures are illustrated in detail for a particular application in Ref. [4]. Attention is being given to equivalent theorems for other models for band absorption.

Acknowledgement. This work was sponsored by the Armed Forces Special Weapons Project through the Department of the Navy, Office of Naval Research, under Contract Nonr-1224(17) with the University of Michigan.

\section{REFERENCES}

1. W. M. Elsasser, Heat transfer by infrared radiation in the atmosphere, Harvard Meteor, Studies No. 6, Harvard Univ., 1942

2. R. Courant and D. Hilbert, Methods of mathematical physics, vol. I, 1st English ed., Interscience Publishers, New York, 1953, p. 93

3. A. Erdelyi, ed., Tables of integral transformations, vol. I, Formula No. 8, McGraw-Hill Book Co., New York, 1953, p. 266

4. J. H. Chin and S. W. Churchill, Radiant heat transfer through the atmosphere, Chem Eng. Progr. Sympos. Series, in press. 